\title{
Linear Programming Bounds for Doubly-Even Self-Dual Codes
}

\author{
Ilia Krasikov and Simon Litsyn, Member, IEEE
}

\begin{abstract}
Using a variant of linear programming method we derive a new upper bound on the minimum distance $d$ of doublyeven self-dual codes of length $n$. Asymptotically, for $n$ growing, it gives $d / n \leq 0.166315 \cdots+o(1)$, thus improving on the Mallows-Odlyzko-Sloane bound of $1 / 6$. To establish this, we prove that in any doubly even-self-dual code the distance distribution is asymptotically upper-bounded by the corresponding normalized binomial distribution in a certain interval.
\end{abstract}

Index Terms - Distance distribution, self-dual codes, upper bounds.

\section{INTRODUCTION}

A SELF-DUAL linear code $C$ of length $n$ and minimum distance $d$ is doubly-even if all its weights are divisible by 4 . It is known that such codes exist only for $n$ divisible by 8 (this result is attributed to Gleason). Let $d_{n}$ be the minimum distance of a doubly-even self-dual code of length $n$. The question is as follows: given $n$, how large $d_{n}$ could be? We consider an asymptotical problem, namely, we want to estimate

$$
\delta=n^{-1} \lim _{n \rightarrow \infty} \sup d_{n} .
$$

We need some notations. In what follows, all logarithms are natural, and the logarithm of a negative number is understood as its real part (by this convention we avoid writing the absolute values of the expressions under logarithms). As usual

$$
H(x)=-x \ln x-(1-x) \ln (1-x)
$$

stands for the natural entropy function. The binomial coefficients are defined by

$$
\left(\begin{array}{l}
x \\
k
\end{array}\right)=\frac{x(x-1) \cdots(x-k+1)}{k !}
$$

where $x$ is arbitrary and $k$ is a nonnegative integer. In particular, for positive $x$

$$
\left(\begin{array}{c}
-x \\
k
\end{array}\right)=(-1)^{k}\left(\begin{array}{c}
x+k-1 \\
k
\end{array}\right) .
$$

Let $\boldsymbol{B}=\left(B_{0}, B_{1}, \cdots, B_{n}\right)$ stand for the distance distribution of a self-dual code $C$. It is invariant under the

Manuscript received November 14, 1995; revised January 2, 1997.

I. Krasikov is with the School of Mathematical Sciences, Tel-Aviv University, Ramat-Aviv 69978 Tel-Aviv, Israel, and Beit-Berl College, Kfar-Sava, Israel.

S. Litsyn is with the Department of Electrical Engineering-Systems, TelAviv University, Ramat-Aviv 69978, Tel-Aviv, Israel.

Publisher Item Identifier S 0018-9448(97)03447-0.
MacWilliams transform

$$
|C| B_{i}=\sum_{j=0}^{n} B_{j} P_{i}(j)
$$

where $P_{i}$ is the corresponding Krawtchouk polynomial of degree $i$

$$
P_{i}(x)=\sum_{k=0}^{i}(-1)^{k}\left(\begin{array}{l}
x \\
k
\end{array}\right)\left(\begin{array}{l}
n-x \\
i-k
\end{array}\right)
$$

(for properties of Krawtchouk polynomials see e.g., [5], [8], [9], [11]).

Self-dual codes attract a great deal of attention, mainly due to their intimate connections with improtant problems in algebra and number theory (see many references in [1], [2], [11], [14]). Most of the results are based on an involved machinery of invariant theory. The following are the best known upper bounds on the minimum distance of doubly-even self-dual codes.

Theorem 1 (Mallows-Sloane): In doubly-even self-dual codes

$$
d \leq 4\lfloor n / 24\rfloor+4 .
$$

An alternative proof of this result will be given in the Appendix. For large $n$, a slightly stronger inequality was established in [13].

Theorem 2 (Mallows-Odlyzko-Sloane): For every constant $b$ there exists an $n_{0}$ such that for $n \geq n_{0}$ in doubly-even self-dual codes

$$
d \leq n / 6-b
$$

Both bounds yield $\delta \leq 1 / 6$. Despite of the general belief that actually $\delta=H^{-1}(1 / 2)=0.110 \ldots$, there was no progress in the last two decades in improving the upper bound of $1 / 6$. For unrestricted self-dual codes the best known upper bound is due to Ward [15] and it also equals $1 / 6$.

In this paper, we obtain an asymptotic improvement of Theorems 1 and 2.

Theorem 3:

$$
\delta \leq c_{\min }
$$

where $c_{\min } \approx 0.166315$, is the only real root of

$$
8 x^{5}-24 x^{4}+40 x^{3}-30 x^{2}+10 x-1 .
$$

To prove it we use a modification of the linear programming method for upper-bounding individual components of the 
distance distribution of codes under consideration. The proof essentially employs estimates for the range of binomiality of codes, the concept introduced in [6], [7]. Roughly speaking, the binomiality means that in a certain range the components of the distance distribution are upper-bounded by the normalized binomial distribution, the same one as of a randomly chosen code. We used the MATHEMATICA package in computations; not all the transformations are straightforward, so we usually present some intermediate results.

\section{BASIC RELATIONS}

Let $C$ be a doubly-even self-dual code. We start with an elementary proof to the result of Gleason.

Theorem 4: $C$ is symmetric, that is, $B_{i}=B_{n-i}$, and $n=0(\bmod 8)$.

Proof: From $|C|=2^{n / 2}$ we deduce that the length $n$ is even. Since $P_{i}(j)=(-1)^{j} P_{n-i}(j)$ and $B_{j}=0$ for $j \neq$ $0(\bmod 4)$, (1) yields that $B_{i}=B_{n-i}$. Hence, $n=0(\bmod 4)$. Indeed, if $n=2(\bmod 4)$, then $B_{n}=0$, contradicting $B_{n}=B_{0}$ and $B_{0}=1$. Now

$$
P_{n / 2}(j)=(-1)^{j / 2}\left(\begin{array}{c}
n \\
n / 2
\end{array}\right)\left(\begin{array}{c}
n / 2 \\
j / 2
\end{array}\right) /\left(\begin{array}{l}
n \\
j
\end{array}\right)
$$

for $j$ even, and $P_{n / 2}(j)=0$ otherwise (see, e.g., [4]). Hence, $P_{n / 2}(j)>0$ if $j=0(\bmod 4)$. Therefore, by (1)

$$
2^{n / 2} B_{n / 2}=\sum_{j=0}^{n} B_{j} P_{n / 2}(j)>0 .
$$

So, if $n=4(\bmod 8)$ then $B_{n / 2}>0$ along with $n / 2 \neq$ $0(\bmod 4)$, a contradiction.

Remark: The last inequality actually shows that in doublyeven self-dual codes $B_{n / 2}$ is the maximal spectral component. To see this just use in (1) the inequality

$$
\left|P_{k}(i)\right| \leq\left|P_{n / 2}(i)\right|
$$

that is valid for $n$ and $i$ even (see [4, Lemma 1]). Noticing, that $P_{n / 2}(j)>0$ for $j=0(\bmod 4)$, we get

$$
2^{n / 2} B_{i}=\sum_{j=0}^{n} B_{j} P_{i}(j) \leq \sum_{j=0}^{n} B_{j} P_{n / 2}(j)=2^{n / 2} B_{n / 2} .
$$

Evidently, the same fact is true for the central component of any code dual to a doubly-even code of even length.

Hence, in what follows we assume everywhere that $n$ is a multiple of 8 .

Let $f(x)$ be a polynomial

$$
f(x)=\sum_{i=0}^{n} A_{i} P_{i}(x)
$$

then (see e.g., [9])

$$
A_{i}=A_{i}(f)=2^{-n} \sum_{j=0}^{n} f(j) P_{j}(i)
$$

in particular

$$
A_{0}(f)=2^{-n} \sum_{j=0}^{n} f(j)\left(\begin{array}{l}
n \\
j
\end{array}\right)
$$

The following lemma is a special case of a proposition due to Delsarte [3].

Lemma 1: Let $f(x)$ be a polynomial of degree $r$

$$
f(x)=\sum_{i=0}^{r} A_{i} P_{i}(x), \quad 0 \leq r \leq n
$$

then

$$
A_{0}|C|+|C| \sum_{i=d}^{r} A_{i} B_{i}=f(0)+f(n)+\sum_{j=d}^{n-d} f(j) B_{j} .
$$

Proof: Calculating $|C| \sum_{i=0}^{r} A_{i} B_{i}$, we get the claim from (1).

Define polynomials

$$
\beta_{h}^{n}(x, k)=\prod_{i=0}^{k-1}\left((n-2 x)^{2}-h^{2} i^{2}\right)
$$

and

$$
\alpha_{h}^{n}(x, k)=x(n-x) \beta_{h}^{n}(x, k) .
$$

The zeros of $\beta_{h}^{n}(x, k)$ are $\frac{n}{2} \pm \frac{h i}{2}, i=0, \cdots, k-1$. The polynomials $\alpha_{h}^{n}(x, k)$ have two extra zeros, 0 and $n$. The choice of the polynomials is motivated by the following immediate consequence of (3).

Lemma 2: Let $k$ be odd and $2 k+2<d$. Then

$$
2^{n / 2} A_{0}\left(\alpha_{8}^{n}(x, k)\right)=2 \sum_{j=d}^{n / 2-4 k} \alpha_{8}^{n}(j, k) B_{j} .
$$

Proof: Degree of $\alpha_{8}^{n}(x, k)$ is $2 k+2$. So, $A_{i}\left(\alpha_{8}^{n}(x, k)\right)=0$ for $i \geq 2 k+3$. Since $k$ is odd and $d$ is divisible by 4 , the sum in the left-hand side of (3) vanishes. Furthermore, $\alpha_{8}^{n}(x, k)=0$ at $x=0, n$ and all $n / 2 \pm 4 i$ where $i=0,1, \cdots, k-1$. The result follows.

We compute $A_{0}\left(\alpha_{8}^{n}(x, k)\right)$ using the values of $A_{0}\left(\beta_{4}^{n}(x, k)\right)$. We start from expanding $\beta_{4}^{n}(x, k)$ in the Krawtchouk basis.

Lemma 3:

$$
\beta_{4}^{n}(x, k)=(2 k) ! \sum_{i=0}^{k} \frac{n-4 i}{n-2 k-2 i}\left(\begin{array}{c}
n / 2-k-i \\
k-i
\end{array}\right) P_{2 i}(x) .
$$

In particular

$$
A_{0}\left(\beta_{4}^{n}(x, k)\right)=(2 k) ! \frac{n}{n-2 k}\left(\begin{array}{c}
n / 2-k \\
k
\end{array}\right) .
$$

Proof: The proof is by induction in $k$. For $k=1$ it is checked directly. Put $y=n-2 x$. The following recurrence holds (see, e.g., [9]):

$$
y^{2} P_{i}(x)=y(i+1) P_{i+1}(x)+y(n-i+1) P_{i-1}(x) .
$$

Substituting

and

$$
y P_{i+1}(x)=(i+2) P_{i+2}(x)+(n-i) P_{i}(x)
$$

$$
y P_{i-1}(x)=i P_{i}(x)+(n-i+2) P_{i-2}(x)
$$


we get

$$
\begin{aligned}
y^{2} P_{i}(x)= & (i+1)(i+2) P_{i+2}(x)+\left(2 n i+n-2 i^{2}\right) P_{i}(x) \\
& +(n-i+1)(n-i+2) P_{i-2}(x)
\end{aligned}
$$

and (replacing $n$ by $2 m$ )

$$
\begin{aligned}
\left(y^{2}-16 k^{2}\right) P_{2 i}(x)= & (2 i+1)(2 i+2) P_{2 i+2}(x) \\
& +\left(8 m i+2 m-8 i^{2}-16 k^{2}\right) P_{2 i}(x) \\
& +(2 m-2 i+1)(2 m-2 i+2) P_{2 i-2}(x) .
\end{aligned}
$$

Using this equality by the induction hypothesis after shifting indices in the sums we get

$$
\begin{aligned}
\beta_{4}^{n}(x, k+1) /(2 k) != & \left(y^{2}-16 k^{2}\right) \beta_{4}^{n}(x, k) /(2 k) ! \\
= & \sum_{i=1}^{k+1} \frac{m-2 i+2}{m-k-i+1}\left(\begin{array}{c}
m-k-i+1 \\
k-i+1
\end{array}\right) \\
& \times 2 i(2 i-1) P_{2 i}(x) \\
& +\sum_{i=0}^{k} \frac{m-2 i}{m-k-i}\left(\begin{array}{c}
m-k-i \\
k-i
\end{array}\right) \\
& \times\left(8 m i+2 m-8 i^{2}-16 k^{2}\right) P_{2 i}(x) \\
& +\sum_{i=0}^{k-1} \frac{m-2 i-2}{m-k-i-1}\left(\begin{array}{c}
m-k-i-1 \\
k-i-1
\end{array}\right) \\
& \times(2 m-2 i-1)(2 m-2 i) P_{2 i}(x) .
\end{aligned}
$$

Routine calculations show that

$$
\begin{aligned}
\beta_{4}^{n}(x, k+1) /(2 k) != & (2 k+2)(2 k+1) \sum_{i=0}^{k+1} \frac{m-2 i}{m-k-i-1} \\
& \times\left(\begin{array}{c}
m-k-i-1 \\
k-i+1
\end{array}\right) P_{2 i}(x)
\end{aligned}
$$

thus proving the claim.

Now we need several combinatorial identities. The next one is a generalization of the known expression for the derivative of Chebyshev polynomials $\cos (t \arccos z)$ [10, p. 258] to noninteger values of $t$.

Lemma 4:

$$
\left.\frac{d^{k} \cos (t \arccos z)}{d z^{k}}\right|_{z=1}=\frac{1}{(2 k-1) ! !} \prod_{i=0}^{k-1}\left(t^{2}-i^{2}\right) .
$$

Proof: The proof is by induction on $k$. For $k=1$ it is checked directly. Denote $F_{t}(z)=F_{t}=\cos (t \arccos z)$. Observe that $F_{t}(z)$ satisfies the following differential equation:

$$
\left(1-z^{2}\right) \frac{d^{2}}{d z^{2}} F_{t}-z \frac{d}{d z} F_{t}+t^{2} F_{t}=0
$$

and is holomorphic at $z=1$. Differentiating this equation $k$ times in $z$ using

$$
\frac{d^{k}}{d z^{k}}(v u)=\sum_{i=0}^{k}\left(\begin{array}{c}
k \\
i
\end{array}\right) \frac{d^{i}}{d z^{i}} v \frac{d^{k-i}}{d z^{k-i}} u
$$

we get

$$
\begin{aligned}
\left(1-z^{2}\right) \frac{d^{k+2}}{d z^{k+2}} F_{t} & -2 k z \frac{d^{k+1}}{d z^{k+1}} F_{t}-2\left(\begin{array}{c}
k \\
2
\end{array}\right) \frac{d^{k}}{d z^{k}} F_{t} \\
& -z \frac{d^{k+1}}{d z^{k+1}} F_{t}-k \frac{d^{k}}{d z^{k}} F_{t}+t^{2} \frac{d^{k}}{d z^{k}} F_{t}=0 .
\end{aligned}
$$

That is, for $z=1$

$$
\left.\frac{d^{k+1}}{d z^{k+1}} F_{t}\right|_{z=1}=\left.\frac{t^{2}-k^{2}}{2 k+1} \frac{d^{k}}{d z^{k}} F_{t}\right|_{z=1} .
$$

Now the induction hypothesis yields the claim.

Lemma 5:

$$
\begin{aligned}
A_{0}\left(\alpha_{h}^{n}(x, k)\right) & =\frac{1}{4} n(n-1) A_{0}\left(\beta_{h}^{n-2}(x, k)\right) \\
& =\left.\frac{h^{2 k} n(n-1)(2 k-1) ! !}{4} \frac{d^{k}}{d z^{k}} \cos ^{n-2} \frac{\theta}{h}\right|_{z=1}
\end{aligned}
$$

where $\theta=\arccos z$.

Proof: Put $s=n-2$.

$$
\begin{aligned}
& A_{0}\left(\alpha_{h}^{n}(x, k)\right) \\
& =2^{-n} \sum_{x=0}^{n} x(n-x)\left(\begin{array}{l}
n \\
x
\end{array}\right) \prod_{i=0}^{k-1}\left((n-2 x)^{2}-h^{2} i^{2}\right) \\
& =\frac{n(n-1)}{2^{n}} \sum_{x=1}^{n-1}\left(\begin{array}{c}
s \\
x-1
\end{array}\right) \prod_{i=0}^{k-1}\left((n-2 x)^{2}-h^{2} i^{2}\right) \\
& =\frac{n(n-1)}{2^{n}} \sum_{x=0}^{s}\left(\begin{array}{l}
s \\
x
\end{array}\right) \prod_{i=0}^{k-1}\left((s-2 x)^{2}-h^{2} i^{2}\right) \\
& =\frac{n(n-1)}{4} A_{0}\left(\beta_{h}^{s}(x, k)\right) \\
& =\frac{h^{2 k} n(n-1)}{2^{n}} \sum_{x=0}^{s}\left(\begin{array}{l}
s \\
x
\end{array}\right) \prod_{i=0}^{k-1}\left(\left(\frac{s-2 x}{h}\right)^{2}-i^{2}\right) \\
& =\left.\frac{h^{2 k} n(n-1)(2 k-1) ! !}{2^{n}} \sum_{x=0}^{s}\left(\begin{array}{l}
s \\
x
\end{array}\right) \frac{d^{k}}{d z^{k}} \cos \frac{s-2 x}{h} \theta\right|_{z=1} \\
& =\frac{h^{2 k} n(n-1)(2 k-1) ! !}{2^{n+1}} \frac{d^{k}}{d z^{k}} \sum_{x=0}^{s}\left(\begin{array}{l}
s \\
x
\end{array}\right) \\
& \times\left.\left(\exp \left(i \frac{s-2 x}{h} \theta\right)+\exp \left(-i \frac{s-2 x}{h} \theta\right)\right)\right|_{z=1} \\
& =\frac{h^{2 k} n(n-1)(2 k-1) ! !}{2^{n+1}} \\
& \times \frac{d^{k}}{d z^{k}}\left(\exp \left(\frac{i s \theta}{h}\right)\left(1+\exp \left(-\frac{2 i \theta}{h}\right)\right)^{s}\right. \\
& \left.+\exp \left(-\frac{i s \theta}{h}\right)\left(1+\exp \left(\frac{2 i \theta}{h}\right)\right)^{s}\right)\left.\right|_{z=1} \\
& =\left.\frac{h^{2 k} n(n-1)(2 k-1) ! !}{2^{n+1}} \frac{d^{k}}{d z^{k}}\left(2^{s+1} \cos ^{s} \frac{\theta}{h}\right)\right|_{z=1} \\
& =\left.\frac{h^{2 k} n(n-1)(2 k-1) ! !}{4} \frac{d^{k}}{d z^{k}} \cos ^{s} \frac{\theta}{h}\right|_{z=1} .
\end{aligned}
$$

Comparing the expressions for $A_{0}\left(\beta_{4}^{n}(x, k)\right)$ in the two previous lemmas we obtain the following corollary.

Corollary 1: For nonnegative integer $\ell$

$$
\left.\frac{d^{k} \cos ^{\ell}\left(\frac{1}{4} \arccos z\right)}{d z^{k}}\right|_{z=1}=\frac{\ell(k-1) !}{2^{3 k+1}}\left(\begin{array}{c}
\frac{\ell}{2}-k-1 \\
k-1
\end{array}\right) .
$$


Lemma 6:

$$
\begin{aligned}
A_{0}\left(\alpha_{8}^{n}(x, k)\right)= & n(n-1)(2 k-1) ! 2^{2 k-n / 2-1} \\
& \times \sum_{j=1}^{n / 2-1} j\left(\begin{array}{c}
n / 2-1 \\
j
\end{array}\right)\left(\begin{array}{c}
j / 2-k-1 \\
k-1
\end{array}\right) .
\end{aligned}
$$

Proof: From the previous lemma

$$
\begin{aligned}
A_{0} & \left(\alpha_{8}^{n}(x, k)\right) \\
= & \left.\frac{8^{2 k} n(n-1)(2 k-1) ! !}{4} \frac{d^{k}}{d z^{k}} \cos ^{n-2} \frac{\theta}{8}\right|_{z=1} \\
= & \left.\frac{8^{2 k} n(n-1)(2 k-1) ! !}{2^{n / 2+1}} \frac{d^{k}}{d z^{k}}\left(1+\cos \frac{\theta}{4}\right)^{n / 2-1}\right|_{z=1} \\
= & \frac{8^{2 k} n(n-1)(2 k-1) ! !}{2^{n / 2+1}} \\
& \times\left.\sum_{j=0}^{n / 2-1}\left(\begin{array}{c}
n / 2-1 \\
j
\end{array}\right) \frac{d^{k}}{d z^{k}} \cos ^{j} \frac{\theta}{4}\right|_{z=1} \\
= & 2^{6 k-n / 2-1} n(n-1)(2 k-1) ! ! \\
& \times \sum_{j=0}^{n / 2-1}\left(\begin{array}{c}
n / 2-1 \\
j
\end{array}\right) \frac{(k-1) !}{2^{3 k+1}} j\left(\begin{array}{c}
j / 2-k-1 \\
k-1
\end{array}\right) \\
= & 2^{2 k-n / 2-1} n(n-1)(2 k-1) ! \\
& \times \sum_{j=0}^{n / 2-1} j\left(\begin{array}{c}
n / 2-1 \\
j
\end{array}\right)\left(\begin{array}{c}
j / 2-k-1 \\
k-1
\end{array}\right) .
\end{aligned}
$$

Actually, we need only odd $k$ 's, so for the sake of simplicity we will formulate all the results below under this assumption. Since our proof of the main theorem consists of several steps and involves a great deal of algebraic manipulations, let us sketch it. First we show that under certain conditions $A_{0}(x, k)>0$, and derive an asymptotic expression for it. Using it we obtain from (6) upper bounds on $B_{j}$ depending on $k$. Optimization in $k$ allows proving that for $\delta>c_{\min }$ the distance distribution components are upper-bounded by the normalized binomial distribution in the range $[\delta n,(1-\delta) n]$. Substituting these bounds into the right-hand side of (6) for a certain choice of $k$ (maximal possible under the conditions of Lemma 2) we get a contradiction.

\section{Bounds ON THE Distance Distribution}

We start with asymptotical evaluation of $A_{0}\left(\alpha_{8}^{n}(x, k)\right)$. Let $\kappa=k / n$.

Lemma 7: Let $k$ be odd, and assume $0 \leq \kappa<\frac{\sqrt{2}}{12}$. Denote

$$
S(j)=j\left(\begin{array}{c}
n / 2-1 \\
j
\end{array}\right)\left(\begin{array}{c}
j / 2-k-1 \\
k-1
\end{array}\right)
$$

and $\eta=j / n$. Then for sufficiently large $n$ the function $|S(\eta n)|$ has two local maxima, one at

$$
\eta_{1}=\frac{1+8 \kappa-\sqrt{1-16 \kappa+128 \kappa^{2}}}{8-16 \kappa}
$$

and another at

$$
\eta_{2}=\frac{1+8 \kappa+\sqrt{1-16 \kappa+128 \kappa^{2}}}{8-16 \kappa} .
$$

The first maximum is the absolute maximum for $\kappa \geq 1 / 12$, otherwise, the second maximum is the absolute one. For $\kappa=1 / 12$ they are asymptotically equal.

Proof: For $k$ odd, $S(j)$ can be negative only for $j$ odd, $j \in J=[2 k+3,4 k-3]$. First, we show that in this interval the maximum of $|S(j)|$ is attained at either end of the interval. To see this consider

$$
r_{j}=\frac{|S(j+2)|}{|S(j)|}=\frac{(j / 2-k)(n / 2-j-1)(n / 2-j-2)}{j(j+1)(2 k-j / 2-1)} .
$$

It is enough to show that there is no $j \in J$ such that $r_{j}>1$ and $r_{j+2}<1$. It is valid if

$$
\begin{aligned}
\frac{d}{d j}((j / 2-k)(n / 2-j-1) & (n / 2-j-2) \\
-j(j+1)(2 k-j / 2-1)) & >0 .
\end{aligned}
$$

The last inequality holds for $\kappa<\frac{\sqrt{2}}{12}$.

Now

$$
\sigma(\eta)=\frac{1}{n} \ln |S(j)|=\frac{1}{2} H(2 \eta)+\left(\frac{\eta}{2}-\kappa\right) H\left(\frac{2 \kappa}{\eta-2 \kappa}\right) .
$$

Differentiating in $j$ we find that there are two maxima stated above, none of them in $J$. Plugging the $\eta_{1}$ and $\eta_{2}$ into (8) we obtain that the corresponding extremal values are

$$
\begin{aligned}
\sigma\left(\eta_{1}\right)= & (1-5 \kappa) \ln 2+\left(\frac{1}{2}-\kappa\right) \ln (1-2 \kappa)-2 \kappa \ln \kappa \\
& -\frac{1}{2} \ln \left(3-16 \kappa+\sqrt{1-16 \kappa+128 \kappa^{2}}\right) \\
& +\kappa \ln \left(1-12 \kappa+128 \kappa^{2}-256 \kappa^{3}\right. \\
& \left.+\sqrt{1-16 \kappa+128 \kappa^{2}}\right)
\end{aligned}
$$

and

$$
\begin{aligned}
\sigma\left(\eta_{2}\right)= & (1-5 \kappa) \ln 2+\left(\frac{1}{2}-\kappa\right) \ln (1-2 \kappa)-2 \kappa \ln \kappa \\
& -\frac{1}{2} \ln \left(3-16 \kappa-\sqrt{1-16 \kappa+128 \kappa^{2}}\right) \\
& +\kappa \ln \left(-1+12 \kappa-128 \kappa^{2}+256 \kappa^{3}\right. \\
& \left.+\sqrt{1-16 \kappa+128 \kappa^{2}}\right) .
\end{aligned}
$$

Now

$$
\begin{aligned}
\sigma\left(\eta_{2}\right)-\sigma\left(\eta_{1}\right)= & \left(\frac{3}{2}-3 k\right) \ln 2+\left(\frac{1}{2}-4 k\right) \ln (1-8 k) \\
& +\left(\frac{1}{2}-k\right) \ln (1-2 k)-k \ln k \\
& -\ln \left(3-16 k-\sqrt{1-16 k+128 k^{2}}\right) \\
& +2 k \ln \left(-1+12 k-128 k^{2}+256 k^{3}\right. \\
& \left.+\sqrt{1-16 k+128 k^{2}}\right) .
\end{aligned}
$$

Furthermore

$$
\begin{aligned}
\frac{d}{d \kappa}\left(\sigma\left(\eta_{2}\right)-\sigma\left(\eta_{1}\right)\right)= & -3 \ln 2-4 \ln (1-8 \kappa)-\ln (1-2 \kappa) \\
& -\ln \kappa+2 \ln \left(-1+12 \kappa-128 \kappa^{2}\right. \\
& \left.+256 \kappa^{3}+\sqrt{1-16 \kappa+128 \kappa^{2}}\right)
\end{aligned}
$$


and we verify that this derivative is strictly negative in the interval $[0, \sqrt{2} / 12]$. For, it is enough to check that

$$
\begin{aligned}
(-1+ & \left.12 \kappa-128 \kappa^{2}+256 \kappa^{3}+\sqrt{1-16 \kappa+128 \kappa^{2}}\right)^{2} \\
& -8(1-8 \kappa)^{4}(1-2 \kappa) \kappa \\
= & 2\left(-1+12 \kappa-128 \kappa^{2}+256 \kappa^{3}\right) \\
& \times\left(-1+12 \kappa-128 \kappa^{2}+256 \kappa^{3}\right. \\
& \left.+\sqrt{1-16 \kappa+128 \kappa^{2}}\right)<0 .
\end{aligned}
$$

Moreover, for $\kappa=1 / 12$ we have $\sigma\left(\eta_{1}\right)=\sigma\left(\eta_{2}\right)$. So, $\sigma\left(\eta_{1}\right)<\sigma\left(\eta_{2}\right)$ for $\kappa<1 / 12$.

Corollary 2: For $k$ odd and $0 \leq \kappa \leq 1 / 12$

$$
\begin{aligned}
\lim _{n \rightarrow \infty} & \frac{1}{n} \ln A_{0}\left(\alpha_{8}^{n}(x, k)\right) \\
= & \left(\frac{1}{2}-\kappa\right) \ln 2+\left(\frac{1}{2}-\kappa\right) \ln (1-2 \kappa)-2 \kappa \\
& -\frac{1}{2} \ln \left(3-16 \kappa-\sqrt{1-16 \kappa+128 \kappa^{2}}\right) \\
& +\kappa \ln \left(-1+12 \kappa-128 \kappa^{2}+256 \kappa^{3}\right. \\
& \left.+\sqrt{1-16 \kappa+128 \kappa^{2}}\right) .
\end{aligned}
$$

Proof: Estimating the sum in the expression for $A_{0}\left(\alpha_{8}^{n}(x, k)\right)$ by the maximum term and using the Stirling approximation for the factorial we get the claim.

\section{Lemma 8:}

$$
\begin{aligned}
\lim _{n \rightarrow \infty} \frac{1}{n} \ln \alpha_{8}^{n}(\iota n, \kappa n)= & 6 \kappa \ln 2+2 \kappa \ln (2 \kappa)-2 \kappa \\
& +\frac{1-2 \iota+8 \kappa}{8} H\left(\frac{16 \kappa}{1-2 \iota+8 \kappa}\right) .
\end{aligned}
$$

Proof: By Stirling approximation.

Now we can show that the distance distribution of self-dual doubly-even codes is upper-bounded in a certain range by the corresponding binomial distribution.

Theorem 5: Let $\iota=\frac{i}{n}$, and $\iota \in[c, 1-c]$, where

$$
c=\frac{1}{2}-\sqrt{\frac{6 \delta-1+\sqrt{1-8 \delta+32 \delta^{2}}}{8(1-\delta)}} .
$$

Then in this interval

$$
\lim _{n \rightarrow \infty} \sup \frac{1}{n} \ln B_{i} \leq H(\iota)-\frac{1}{2} .
$$

Proof: We will prove the theorem by varying the degree of $\alpha_{8}^{n}(x, k)$. If $k$ is odd, $2 k+2<d$, and $d \leq i \leq \frac{n}{2}-4 k$, then by Lemma (2)

$$
B_{i} \leq 2^{n / 2-1} \frac{A_{0}\left(\alpha_{8}^{n}(x, k)\right)}{\alpha_{8}^{n}(i, k)} .
$$

Indeed, $\alpha_{8}^{n}(i, k)>0$ for such $k$ 's.

Choose

$$
\kappa=\frac{(1-2 \iota)^{2}\left(\iota^{2}+(1-\iota)^{2}\right)}{8\left(\iota^{4}+(1-\iota)^{4}\right)} .
$$

Direct checking shows that for $\iota \in\left[c_{1}, 1-c_{1}\right]$ we have $\kappa \leq \frac{1}{12}$, where $c_{1}$ is the smallest real root of the equation

$$
20 x^{4}-40 x^{3}+30 x^{2}-10 x+1=0
$$

$c_{1} \approx 0.16563 \ldots$ Since $c(\delta)$ is decreasing in $\delta$, the minimum $c_{\min }$ of $c(\delta)$ under the condition $c(\delta) \geq \delta$ is determined by the equation $c(\delta)=\delta$ and thus is the only real root of the equation

$$
8 x^{5}-24 x^{4}+40 x^{3}-30 x^{2}+10 x-1=0 .
$$

Notice that for $\delta_{\min }=c_{\min }$ we have $2 \kappa\left(\delta_{\min }\right)=\delta_{\min }$ since the equation $c(\delta)=\delta$ is equivalent to $2 \kappa(\delta)=\delta$. Numerically, $c_{\min }=0.166315 \ldots$ Hence, $c_{1}<c_{\min }$, and $\kappa<1 / 12$.

Furthermore, since the $\kappa$ chosen is decreasing in $\iota$, to validate the condition $2 k+2<d$ we need $2 \kappa<\iota$ in the interval $[c, 1-c]$. The four roots of the equation $2 \kappa=\iota$, are

$$
\frac{1}{2} \pm \sqrt{\frac{6 \delta-1 \pm \sqrt{1-8 \delta+32 \delta^{2}}}{8(1-\delta)}} .
$$

The following two

$$
c_{1,2}=\frac{1}{2} \pm \sqrt{\frac{6 \delta-1+\sqrt{1-8 \delta+32 \delta^{2}}}{8(1-\delta)}}
$$

are real, and $c_{1}+c_{2}=1$. Therefore, for $c$ being the smaller one we conclude that $2 \kappa<\delta$ whenever $i \in[c, 1-c]$.

Now, using (11) and (10) and for the $\kappa$ chosen we obtain the claim from the previous corollary.

Theorem 6: Let $c_{\min }$ be the only real root of

$$
8 x^{5}-24 x^{4}+40 x^{3}-30 x^{2}+10 x-1 .
$$

If there exists a doubly-even self-dual code with $\delta \geq$ $c_{\min } \approx 0.166315$, then all its spectrum is asymptotically upper-bounded by the corresponding normalized binomial distribution.

Proof: It can be checked directly that under the condition of the corollary $c$, defined in the previous theorem, is less than $\delta$.

\section{Proof of the Main Theorem}

By Theorem 1 we can assume that $c_{\min }<\delta \leq 1 / 6$. Choose in Lemma 2 the largest possible odd $k=(d-6) / 2$. That is, $\kappa=\delta / 2$. We have for the left-hand side of (6), by (9)

$$
\begin{aligned}
L= & \lim _{n \rightarrow \infty} \sup \frac{1}{n} \ln \left(2^{n / 2} A_{0}\left(\alpha_{8}^{n}(x,(d+2) / 2)\right)\right) \\
= & -\delta+\left(1-\frac{\delta}{2}\right) \ln 2+\frac{1-\delta}{2} \ln (1-\delta) \\
& -\frac{1}{2} \ln \left(3-8 \delta-\sqrt{1-8 \delta+32 \delta^{2}}\right) \\
& +\frac{\delta}{2} \ln \left(-1+6 \delta-32 \delta^{2}+32 \delta^{3}+\sqrt{1-8 \delta+32 \delta^{2}}\right) .
\end{aligned}
$$

Now, by Theorem 6, to upper-bound the right-hand side of (6) we can substitute the upper binomial estimates of $B_{j}$ 's. This gives by virtue of (10)

$$
\begin{aligned}
R & \left.=\lim _{n \rightarrow \infty} \sup \frac{2}{n} \sum_{j=d}^{n / 2-2 d-4} \alpha_{8}^{n}(j,(d+2) / 2)\right) B_{j} \\
& \leq \max _{\eta \in[\delta, 1 / 2-2 \delta]} u(\eta)
\end{aligned}
$$


where

$$
\begin{aligned}
u(\eta)= & 3 \delta \ln 2+\delta \ln \delta-2 \delta \\
& +\frac{1-2 \eta+4 \delta}{8} H\left(\frac{8 \delta}{1-2 \eta+4 \delta}\right)+H(\eta)-\frac{1}{2} .
\end{aligned}
$$

So, the inequality $L-R \leq 0$ should hold. In what follows, we will show that for $\delta \in\left(c_{\min }, 1 / 6\right]$ this is not true, and thus such a code does not exist.

First we show that

$$
\max _{\eta \in[\delta, 1 / 2-2 \delta]} u(\eta)=u(\delta) .
$$

By differentiation, we obtain

$$
u^{\prime}=\frac{d u(\eta)}{d \eta}=\frac{1}{4} \ln \frac{1-4 \delta-2 \eta}{1+4 \delta-2 \eta}+\ln \frac{1-\eta}{\eta} .
$$

Observe that $u^{\prime}<0$ for $\delta>c_{\min }$. Indeed, this is equivalent to

$$
\delta>\frac{1-6 \eta+4 \eta^{2}-16 \eta^{3}+8 \eta^{4}}{4\left(\eta^{4}+(1-\eta)^{4}\right)} .
$$

The right-hand side of this inequality is a decreasing function in $\eta$, so we check it for $\eta=\delta$. The inequality holds precisely for $\delta>c_{\text {min }}$. Hence

$$
\begin{aligned}
R \leq & u(\delta) \\
\leq & \left(3 \delta-\frac{1}{2}\right) \ln 2+\delta(\ln \delta-1) \\
& +\frac{1+2 \delta}{8} H\left(\frac{8 \delta}{1+2 \delta}\right)+H(\delta)
\end{aligned}
$$

and

$$
\begin{aligned}
L-R= & (12 \ln 2-4 \delta \ln 2+\ln (1-6 \delta)-6 \delta \ln (1-6 \delta) \\
& +12 \ln (1-\delta)-12 \delta \ln (1-\delta)+8 \delta \ln \delta \\
& -\ln (1+2 \delta)-2 \delta \ln (1+2 \delta) \\
& -4 \ln \left(3-8 \delta-\sqrt{1-8 \delta+32 \delta^{2}}\right) \\
& +4 \delta \ln \left(1-6 \delta+32 \delta^{2}\right. \\
& \left.\left.-32 \delta^{3}-\sqrt{1-8 \delta+32 \delta^{2}}\right)\right) / 8
\end{aligned}
$$

$$
\begin{aligned}
\frac{d}{d \delta}(L-R)= & (-2 \ln 2-3 \ln (1-6 \delta)-6 \ln (1-\delta)+4 \ln \delta \\
& -\ln (1+2 \delta)+2 \ln \left(-1+6 \delta-32 \delta^{2}+32 \delta^{3}\right. \\
& \left.\left.+\sqrt{1-8 \delta+32 \delta^{2}}\right)\right) / 4 .
\end{aligned}
$$

One can check that $c_{\min }$ is a root of $\frac{d}{d \delta}(L-R)$. This is equivalent to $c_{\min }$ being a root of

$$
\begin{aligned}
\left(-1+6 \delta-32 \delta^{2}+32 \delta^{3}\right. & \left.+\sqrt{1-8 \delta+32 \delta^{2}}\right)^{2} \delta^{4} \\
& -4(1-6 \delta)^{3}(1-\delta)^{6}(1+2 \delta)=0 .
\end{aligned}
$$

The equation can be transformed into

$$
\begin{aligned}
16 & (1-\delta)^{2}\left(1-10 \delta+30 \delta^{2}-40 \delta^{3}+24 \delta^{4}-8 \delta^{5}\right) \\
& \times\left(1-32 \delta+415 \delta^{2}+2714 \delta^{3}\right. \\
& -8515 \delta^{4}+4052 \delta^{5}+52448 \delta^{6}-143768 \delta^{7} \\
& +91456 \delta^{8}+208576 \delta^{9}-492328 \delta^{10} \\
& \left.+466816 \delta^{11}-238048 \delta^{12}+59168 \delta^{13}\right)=0
\end{aligned}
$$

giving the result.
Moreover, as it is easy to check, for $\delta \leq 1 / 6$, that

$$
\begin{aligned}
& \frac{d^{2}}{d \delta^{2}}(L-R) \\
& =(12 \ln 2+\ln (1-6 \delta)+12 \ln (1-\delta)-\ln (1+2 \delta) \\
& \left.\quad-4 \ln \left(3-8 \delta-\sqrt{1-8 \delta+32 \delta^{2}}\right)\right) / 8>0 .
\end{aligned}
$$

Thus $c_{\min }$ is the only root of $\frac{d}{d \delta}(L-R)$ in the interval under consideration. It remains to prove that $L-R=0$ for $\delta=c_{\min }$. Indeed, consider the function

$$
\begin{aligned}
\rho(\delta)= & (L-R)-\delta \frac{d}{d \delta}(L-R) \\
= & (12 \ln 2+\ln (1-6 \delta)+12 \ln (1-\delta)-\ln (1+2 \delta) \\
& \left.-4 \ln \left(3-8 \delta-\sqrt{1-8 \delta+32 \delta^{2}}\right)\right) / 8 .
\end{aligned}
$$

Now, $\rho(\delta)=0$ is equivalent to

$$
2^{12}(1-6 \delta)(1-\delta)^{12}-(1+2 \delta)\left(3-8 \delta-\sqrt{1-8 \delta+32 \delta^{2}}\right)^{4}=0
$$

or, getting rid of the square root

$$
\begin{aligned}
64 & (1-\delta)^{4}\left(1-10 \delta+30 \delta^{2}-40 \delta^{3}+24 \delta^{4}-8 \delta^{5}\right) \\
& \times\left(3825-86370 \delta+862866 \delta^{2}-5181544 \delta^{3}\right. \\
& +21170188 \delta^{4}-62816720 \delta^{5}+140812600 \delta^{6} \\
& -244608448 \delta^{7}+334412032 \delta^{8}-362393120 \delta^{9} \\
& +311228224 \delta^{10}-210349056 \delta^{11}+110332288 \delta^{12} \\
& -43912192 \delta^{13}+12798976 \delta^{14}-2574848 \delta^{15} \\
& \left.+319488 \delta^{16}-18432 \delta^{17}\right)=0
\end{aligned}
$$

proving the claim.

Hence, finally, $L-R>0$ for $\delta \in\left(c_{\min }, 1 / 6\right]$, a contradiction.

\section{APPENDIX}

Here we sketch a proof of Theorem 1. In contrast to the original proof we use only properties of the MacWilliams transform.

The following auxiliary lemma is used.

Lemma 9: If $A_{0}\left(\alpha_{8}^{n}(x, k)\right) \neq 0$, for some $k$, then $d \leq$ $\max \left\{2 k+2, \frac{n}{2}-4 k\right\}$.

Proof: Note that $\alpha_{8}^{n}(x, k)=0$ at $x=0, n$, and all $n / 2 \pm 4 i$, where $i=0,1, \cdots, k-1$. Assume that for $k$ chosen $A_{0}\left(\alpha_{8}^{n}(x, k)\right) \neq 0$. Plugging $\alpha_{8}^{n}(x, k)$ into (3), we get that either

i) $\sum_{j=d}^{n-d} \alpha_{8}^{n}(j, k) B_{j}=2 \sum_{j=d}^{n / 2-4 k} \alpha_{8}^{n}(j, k) B_{j} \neq 0$

or

ii) $\sum_{i=d}^{2 k+2} A_{i} B_{i} \neq 0$

If i) holds, then $n / 2-4 k \geq d$. If ii) is true, then $2 k+2 \geq d$.

Now we are ready to prove Theorem 1 . 
Proof: We consider three cases, depending on $n$ modulo 24. In all these cases we prove that $A_{0}\left(\alpha_{8}^{n}(x, k)\right)>0$. Namely, referring to Lemma 9

if $n=0(\bmod 24)$ we choose $k=n / 12+1$, giving

$d \leq \frac{n}{6}+4$

if $n=8(\bmod 24)$ we choose $k=(n+4) / 12$, giving

$d \leq \frac{n+16}{6}$

if $n=16(\bmod 24)$ we choose $k=(n-4) / 12$, giving $d \leq \frac{n+8}{6}$.

Observe, that all the chosen $k$ 's are odd. Then using the same arguments as in the proof of Lemma 7 we demonstrate that in the expression for $A_{0}\left(\alpha_{8}^{n}(x, k)\right)$ there is a positive dominating summand. To prove this for all $n$ use the Stirling approximation

$$
\begin{aligned}
& \frac{1}{2} \log \frac{\pi}{4}-\frac{1}{2} \log \frac{2 \pi i(n-i)}{n}+n H\left(\frac{i}{n}\right) \\
&<\log \left(\begin{array}{c}
n \\
i
\end{array}\right)<-\frac{1}{2} \log \frac{2 \pi i(n-i)}{n}+n H\left(\frac{i}{n}\right) .
\end{aligned}
$$

It proves the claim for $n>100$. The small cases (there are only 12 such lengths) are checked directly.

\section{ACKNOWLEDGMENT}

The authors wish to thank V. Levenshtein, P. Solé, and the anonymous referees for useful comments and suggestions.

\section{REFERENCES}

[1] J. H. Conway and N. J. A. Sloane, Sphere Packings, Lattices and Groups. Berlin, Germany: Springer-Verlag, 1988.

[2] J. H. Conway and N. J. A. Sloane, "A new upper bound on the minimum distance of self-dual codes," IEEE Trans. Inform. Theory, vol. 36, pp. 1319-1333, Nov. 1990.

[3] P. Delsarte, An Algebraic Approach to the Association Schemes of Coding Theory (Philips Res. Rep. Suppl.), no. 10, 1973.

[4] I. Krasikov and S. Litsyn, "On spectra of BCH codes," IEEE Trans. Inform. Theory, vol. 41, pp. 786-788, May 1995.

[5] _ "On integral zeros of Krawtchouk polynomials," J. Comb. Theory (Ser. A), vol. 74, no. 1, pp. 71-99, 1996.

[6] _ "Bounds on spectra of codes with known dual distance," Des. Codes Cryptogr., to be published.

[7] ___ "Estimates for the range of binomiality in codes' spectra," IEEE Trans. Inform. Theory, vol. 43, pp. 987-991, May 1997.

[8] V. I. Levenshtein, "Krawtchouk polynomials and universal bounds for codes and designs in Hamming spaces," IEEE Trans. Inform. Theory, vol. 41, pp. 1303-1321, Sept. 1995.

[9] J. H. van Lint, Introduction to Coding Theory. Berlin, Germany: Springer-Verlag, 1992.

[10] W. Magnus, F. Oberhettinger, and R. P. Soni, Formulas and Theorems for the Special Functions of Mathematical Physics, 3rd ed. New York/Berlin: Springer-Verlag, 1966.

[11] F. J. MacWilliams and N. J. A. Sloane, The Theory of Error-Correcting Codes. New York: North-Holland, 1977.

[12] C. L. Mallows and N. J. A. Sloane, "An upper bound for self-dual codes," Inform. Contr., vol. 22, pp. 188-200, 1973.

[13] C. L. Mallows, A. M. Odlyzko, and N. J. A. Sloane, "Upper bounds for modular forms, lattices, and codes," J. Alg., vol. 36, pp. 68-76, 1975.

[14] V. Pless, Introduction to the Theory of Error-Correcting Codes. New York: Wiley, 1982

[15] H. N. Ward, "A bound for divisible codes," IEEE Trans. Inform. Theory, vol. 38, pp. 191-194, Jan. 1992. 